urgent inpatient colonoscopy. Average time till patients received urgent inpatient endoscopy was 2.88 days. Of 15 patients who required transfusion, 12 were correctly transfused. $57.80 \%$ of patients had warfarin or DOAC stopped at presentation. $5.26 \%$ of patients had anticoagulation correctly restarted following haemostasis. Similarly $40.00 \%$ of patients had aspirin stopped at presentation and $0.00 \%$ and $15.38 \%$ of patients had aspirin restarted correctly for primary and secondary prevention respectively.

Conclusions Auditing against a new standard has revealed worrying data and highlights the importance of change to practice that these guideline provide. This is best seen with the improper management of patients on anticoagulant and antiplatelet agents. However, the guideline is arguably overly cautious and strict adherence would place significant strain on a DGH. We propose several amendments to the guideline such as redefining admission criteria and the approach to managing unstable LGIB. Our modified guideline shows minimum expected clinical practice that is conducive to high quality patient care within the limits of hospital resources.

\section{P388 COGNITIVE IMPAIRMENT PREDICTS MORTALITY AND LONGER ADMISSIONS FOR INDEX PRESENTATIONS OF ALCOHOL-RELATED LIVER DISEASE}

${ }^{1,2}$ Anahita Sharma* ${ }^{1}$ Constantinos Kallis, ${ }^{1}$ Pete Dixon, ${ }^{1,2}$ Benjamin Silberberg, ${ }^{2}$ Steve Hood,
${ }^{1,2}$ Keith Bodger. ' University of Liverpool, Liverpool, UK; ${ }^{2}$ Aintree University Hospital,
Liverpool University Hospitals NHS Foundation Trust, Fazakerley, Liverpool, UK 10.1136/gutjnl-2020-bsgcampus.462

Introduction Alcohol-related liver disease (ARLD) can present with neuropsychiatric complications. Epidemiological studies have not investigated the impact of confusional states on patient journeys. As part of the Connected Health Cities programme, we retrospectively investigated coding data from a regional administrative dataset to identify the burden of recognised cognitive impairment (CI) in a pre-identified cohort of patients during their index admission of ARLD (fiscal years 2014-18).

Methodology Inpatient spells for a cohort of 3,887 index ARLD admissions were screened for ICD-10 codes indicative of acute and chronic CI. Descriptive analytics and stepwise multivariate logistic regression models were generated using Stata 15 (StataCorp, 2017) for predefined outcomes: inpatient mortality, length of stay, all-cause outpatient attendance and all-cause A\&E attendance. These were casemix-adjusted for age, sex, co-morbidity, deprivation and variables associated with severity of liver decompensation.

Results 20 codes corresponded to acute or chronic CI, most frequently encoding encephalopathy and alcohol-related amnesiac syndrome respectively. Codes for intoxication and withdrawal were excluded and adjusted for. 277 spells (7.1\%) were coded with $\geq 1$ ICD code for acute CI, and $78(2.0 \%)$ for chronic CI, with minimal overlap (0.6\%). Comparisons were made with patients without relevant codes. Overall, these patients were older (mean age 57.5 and 64.1 respectively) with higher levels of co-morbidity (mean Charlson index 16.4 and 12.6 respectively) with median bed-days of 13 and 14 . Multivariate logistic regression models demonstrated patients with acute CI had higher odds of inpatient mortality (OR 2.13) and long admission $\geq 21$ days (OR 2.30). Patients coded with chronic CI had higher odds of long admission (OR 3.09) and A\&E attendance within 90 days (OR 2.01), and were less likely to attend an outpatient clinic at 14-day (OR 0.28) and 30-day (OR 0.4) intervals post-discharge. CI did not predict risk of readmission.

Conclusion In terms of mortality, acute CI is likely to reflect higher disease severity, particularly as encephalopathy is a poor prognosticator in this group of patients. The evidence suggests that chronic CI in patients with ARLD is underdetected in clinical practice. Our analysis demonstrates patients with recognised chronic CI experience extended admissions, are more likely to experience unplanned care and less likely to engage with outpatient care up to 90 days post-discharge. This suggests that current care models may not be appropriate for this subgroup, and alternative pathways integrating prompt identification and supported discharge mechanisms should be developed.

\section{P389 VAGUE ABDOMINAL SYMPTOMS PATHWAY: IS IT WORTH IT?}

${ }^{1}$ Panagiotis Stamoulos*, ${ }^{1}$ Ketul Patel, ${ }^{1}$ Robyn Jenkins, ${ }^{1}$ Nicola Beech, ${ }^{1}$ Claire Walters, 1,2Jennifer Ross, 'Sanjay Gupta. 'Croydon University Hospital, London, UK; ${ }^{2}$ St George's Hospital, London, UK

\subsection{6/gutjnl-2020-bsgcampus.463}

Introduction The time to diagnosis for patients with suspected cancer in the NHS is often excessive leading to unnecessary distress for patients and contributing to the poor survival rates in the UK compared to Europe. The Accelerate, Coordinate, Evaluate (ACE) Programme is an early diagnosis of cancer initiative focused on testing innovations that either identify individuals at high risk of cancer earlier or streamline diagnostic pathways.

Croydon University Hospital was chosen as one of three hospital sites across West London to participate in this pilot project. The aim was to implement and evaluate a vague abdominal symptoms (VAS) referral pathway from general practice to acute oncology within Croydon in order to reduce emergency admissions and late presentation of cancer.

Methods The pathway was implemented from April 2017 to March 2018. The pathway required GP practices to refer according to defined eligibility criteria. All referrals were triaged by an Acute Oncology Nurse specialist and reviewed in a dedicated gastroenterology clinic. All patients underwent initial investigations within 14 days of clinic review. Virtual clinic and feedback was provided to GPs and patients after completion of investigations.

Results 60 patients were referred of which 51 were assessed on the pathway ( 7 did not meet eligibility criteria and 2 refused to be seen) with a median age of 56 years. Commonest referral indication was abdominal pain (73\%).33\% of patients had visited their GP at least three times for symptoms meeting the eligibility criteria of the pathway.

$34(67 \%)$ patients had endoscopy and 41 (80\%) had radiology investigations. $23(45 \%)$ of patients had more than 3 tests. Metastatic cancer was detected in 2 patients-splenic sarcoma and squamous lung carcinoma. 15 (29\%) of patients had significant benign conditions including mesenteric ischaemia, Crohn's disease, granulomatous gastritis, intraductal papillary mucinous neoplasm, lymphocytic enteritis, gallstones, gallbladder polyps, colonic polyps and hydrosalpinx. 34 (67\%) patients had other benign conditions. Median time to final diagnosis was 57 days (the 2 cancers were diagnosed at 7 and 34 days). 